\title{
Plants and gall hosts of the Tirimbina Biological Reserve, Sarapiqui, Costa Rica: Combining field sampling with herbarium records
}

\author{
Juan Manuel Ley-López ${ }^{1}$, José González² \& Paul E. Hanson ${ }^{3 *}$ \\ 1. Departamento Académico, Reserva Biológica Tirimbina. Sarapiquí, Heredia, Costa Rica; juanmacrbiol@gmail.com \\ 2. Independent consultant, Costa Rica; jagragave@gmail.com \\ 3. Escuela de Biología, Universidad de Costa Rica; San Pedro, 11501-2060 San José, Costa Rica; \\ phanson91@gmail.com \\ * Correspondence
}

Received 03-X-2018. Corrected 10-I-2018. Accepted 24-I-2019.

\begin{abstract}
There has been an increasing number of inventories of gall-inducing arthropods in the Neotropics. Nonetheless, very few inventories have been carried out in areas where the flora is well documented, and records of galls from herbaria and sites outside the study area have seldom been utilized. In this study we provide a checklist of the native vascular plants of a 345 ha forest reserve in the Caribbean lowlands of Costa Rica and document which of these plants were found to harbor galls. The gall surveys were carried out between November 2013 and December 2016. We also cross-checked our plant list with the previous gall records from elsewhere in the country and searched for galls on herbarium specimens of dicots reported from the reserve. In total, we recorded 143 families and 1174 plant species, of which 401 were hosts of galls. Plant hosts of galls were found in the following non-mutually exclusive categories: 209 in our field sampling, 257 from previous records, and 158 in herbarium specimens. Of our field records of galls, 77 were new for the country, 77 were also found in the herbarium and 110 had been previously recorded in the country. Herbarium specimens and previous records are good indicators of potential gall hosts but around one quarter (40/158) of the gall records in the herbarium were ambiguous as to whether the tissue alteration was a gall or not. Plant family size was related to number of gall hosts, but most dicot families have similar proportions of gall host species. We concluded that more than a third of the native plants in the reserve are gall hosts, but this number increased to $44.93 \%$ when only dicots were considered. Our results demonstrate the utility of providing complete plant inventories when conducting gall inventories, and of using herbarium specimens and previous galls studies to infer gall diversity in a specific area.
\end{abstract}

Key words: Cecidomyiidae; checklist; floristic composition; gall diversity; inventories.

Ley-López, J. M., González, J., \& Hanson, P. H. (2019). Plants and gall hosts of the Tirimbina Biological Reserve, Sarapiqui, Costa Rica: Combining field sampling with herbarium records. Revista de Biología Tropical, 67(2) Suplemento, S212-S227.

Gall-inducing insects have been called "nature's most sophisticated herbivores" due to their ability to stimulate atypical plant growths that provide the insect with both food and shelter (Shorthouse, Wool, \& Raman, 2005). The mechanisms by which galls are induced are poorly understood, although this topic has received considerable attention in a species that is a pest of wheat (Stuart, Chen, Shukle, \&
Harris, 2012) as well as various gall-inducers in Brazil (Oliveira et al., 2016). In addition to species in six orders of insects, certain mites (especially Eriophyidae) also induce plant galls (Raman, Schaefer, \& Withers, 2005). The majority of these gall-inducing arthropods are highly host-specific (Butterill \& Novotny, 2015) and each species of gall-inducer produces a diagnostic gall morphology (Isaias, 
Carneiro, Oliveira, \& Santos, 2013). Because most gall-inducing arthropods are undescribed species, gall inventories are usually based on a combination of plant identification and gall morphotyping (Espirito-Santo \& Fernandes, 2007; Carneiro et al., 2009), the latter serving as an extended phenotype of the gall-inducer (Carneiro, Pacheco, \& Isaias, 2015).

There have been an increasing number of inventories of neotropical galls during the last two decades (Gagné, 1994; Hanson \& Gómez-Laurito, 2005; Fernandes, Coelho \& Santos, 2014; Maia \& Mascarenhas, 2017). Gall-inducers appear to be less diverse on ferns, gymnosperms and monocots (Gagné, 1994; Santos, Hanson, Maia, \& Mehltreter, 2019). In contrast, it has been suggested that as many as $90 \%$ of dicots could harbor gallinducing cecidomyiids, which is probably the most diverse group of gall-inducers (Hanson \& Gómez-Laurito, 2005). Studies have shown that certain plant families and genera have a particularly rich fauna of gall-inducing arthropods (Araújo, Santos, \& Gomes-Klein, 2012), a trend that is correlated to taxon size (Fernandes, 1992; Araújo, 2011; Araújo et al., 2012). In addition, some plant species have been referred to as "superhosts" due to their relatively high species richness of gall-inducers (Fernandes et al., 1996).

Despite the increasing number of gall inventories, comparisons between surveyed areas are problematic since different sampling efforts and methodologies have been used. Furthermore, a thorough inventory of gallinducers, even at a single site, is fraught with difficulties (Hanson \& Gómez-Laurito, 2005): many galls are hidden inside fruits and other plant organs (or in the roots), galls in the canopy are generally inaccessible (but see Julião, Venticinque, Fernandes, \& Price, 2014), not all individuals of a plant species harbor galls, and some galls are seasonal. In Costa Rica there have been extensive surveys of both plants (Hammel, Grayum, Herrera, \& Zamora, 20032015) and gall-inducers (Hanson \& GómezLaurito, 2005), yet there are no intensive gall inventories for particular sites. Therefore, a major aim of the present investigation was to provide an inventory of the galls for a 345 ha reserve in the lowlands of Costa Rica.

In addition to providing the first gall inventory of a particular site in Costa Rica, the present investigation also included two components that, to the best of our knowledge, have never been included in previous inventories. First, in addition to inventorying the galls and their host plants, we also inventoried the vascular plants of the site, thereby including negative records. Second, by conducting a complete plant inventory, we were able to broaden the survey to include plant-gall records from other parts of the country as well as from herbarium samples.

\section{MATERIALS AND METHODS}

Study site: Fieldwork was carried out at the Tirimbina Biological Reserve (TBR, $10^{\circ} 25^{\prime}$ N \& $84^{\circ} 47^{\prime}$ W), Sarapiqui, Heredia, Costa Rica. This site is considered a humid tropical wet forest (Holdridge, 1978), between 180 and $220 \mathrm{~m}$ a.s.l., with an average precipitation and temperature of $3777 \mathrm{~mm}$ and $24.3{ }^{\circ} \mathrm{C}$, respectively. The reserve consists of 345 ha, mainly of mature forest, and is bordered on its western edge by the Sarapiqui River; there was occasional selective logging between 1962 and 1990 . The reserve maintains a trail system of ca. $10 \mathrm{~km}$. TBR is located $17 \mathrm{~km}$ from La Selva Biological Station (belonging to the Organization for Tropical Studies), one of the best sampled areas in the Neotropics in terms of plants (see, http://sura.ots.ac.cr/florula4/).

Plant inventory: The principal plant inventory was carried out by JG under a consultancy paid by the TBR from June 2008 to October 2009. During this period around 75 field samplings, comprising on average $4 \mathrm{~h}$ each, were done by walking the trails in the reserve. All vascular plants observed and identified in the field were annotated in the list. Doubtful species were collected to be identified later in the herbarium and fertile individuals were collected to provide herbarium specimens. In total, 1213 species were identified during this period 
of which 1131 samples belonging to 657 species were deposited at the La Selva Biological Station Herbarium (LSCR). We excluded records of non-native plants in the reserve since galls are unlikely to occur on these species. Scientific names were revised using The Taxonomic Name Resolution Service (2018) with data derived from Tropicos (2018) and USDA (2018) plant databases. Although we recognize that "dicots" represent a paraphyletic group, we use the term for convenience.

Gall inventory: Galls were sampled by three methods. From November 2013 to December 2016 a general sampling was carried out by haphazardly walking the trails and examining the vegetation along the pathways at least twice a week for an average of $2 \mathrm{~h}$ per sampling period. Second, to complement the first method, galls were collected opportunistically throughout the study period during the course of other research projects in the reserve. Third, from August to October 2016, 22 random transects of $50 \times 2 \mathrm{~m}$ were performed across the reserve in which every plant was sampled. The main goal of the latter was to assess the effectiveness of previous sampling. Galls were collected primarily from leaves and stems; roots, flowers and fruits were generally not examined (e.g. all native figs have galls in the syconia, but these were not included).

Samples of galls were collected in plastic bags for later examination in the laboratory while plants were identified in situ or, when necessary, plant samples were collected for later identification. Plant identifications benefitted from the previous plant list that had been prepared for the reserve. Whenever possible, a subset of galls was dissected under a microscope in order to attempt to determine the family-level identity of the gall-inducer, although this was not the primary focus of the present investigation and therefore only a general summary of the gall-inducers is provided here.

Two additional sources of data were utilized in order to provide a more complete inventory of the galls. First, for plant species present in the TBR we added records (where available) of galls from the same plant species collected elsewhere in the country during the last 25 years (Hanson, unpublished data). Second, during 2017 we searched for galls on specimens at the University of Costa Rica Herbarium (USJ), examining all dicot species reported from the reserve; dicots were chosen because galls are much less common on other groups of vascular plants (Hanson \& GómezLaurito, 2005). We examined every sample found in the herbarium (regardless of locality in Costa Rica) although for species with a very large number of specimens only the two folders with the most specimens were examined. All samples with signs of galls were observed under the microscope and then assigned to one of three categories: probably not a gall (these records were excluded), almost certainly a gall, or questionably a gall (these records are indicated by a question mark in the results). It is generally impossible to determine the identity of the gall-inducer based on herbarium specimens (except perhaps in the case mite erinea - hyperplasia of plant trichomes in response to mite activity), so this was not attempted, nor was this the focus of the present investigation.

\section{RESULTS}

Plant diversity: A total of 1174 native plant species belonging to 143 families were found in the Tirimbina Biological Reserve (Tables 1-2). Pteridophytes (ferns and their allies) were represented by 122 species, while gymnosperms were represented by only two species. Angiosperms were represented by 242 species of monocots and 808 of dicots (Table 1). The six most species-rich families, in descending order, were Rubiaceae (79), Fabaceae (67), Araceae (66), Melastomataceae (57), Asteraceae (39) and Piperaceae (37). Seven additional families were represented by 20 or more species each and 24 were represented by 10-19 species. On the other hand, 35 families were represented by only one species (Table 2 ).

Gall diversity: In total, we found 401 plant species that hosted galls, representing 
TABLE 1

Numbers of gall host plants in the Tirimbina Biological Reserve found by: field sampling (FS), records from the herbarium $(\mathrm{H})$, and previous records from elsewhere in the country $(\mathrm{PR})$

\begin{tabular}{|c|c|c|c|c|c|}
\hline & Ferns & Gymnosperms & Monocots & Dicots & Total \\
\hline No record & 115 & 2 & 211 & 445 & 773 \\
\hline FS & 2 & 0 & 10 & 65 & 77 \\
\hline $\mathrm{H}$ & NA & NA & NA & 45 & 45 \\
\hline PR & 4 & 0 & 13 & 94 & 111 \\
\hline $\mathrm{FS}+\mathrm{H}$ & NA & NA & NA & 22 & 22 \\
\hline $\mathrm{FS}+\mathrm{PR}$ & 1 & 0 & 8 & 46 & 55 \\
\hline $\mathrm{H}+\mathrm{PR}$ & NA & NA & NA & 36 & 36 \\
\hline $\mathrm{FS}+\mathrm{H}+\mathrm{PR}$ & NA & NA & NA & 55 & 55 \\
\hline Total (galls) & 7 & 0 & 31 & 363 & 401 \\
\hline Total (plants) & 122 & 2 & 242 & 808 & 1174 \\
\hline
\end{tabular}

$\mathrm{NA}=$ not applicable (only dicots were examined in the herbarium).

$34.16 \%$ of the flora of the reserve (Table 1 ). When considering only dicots this percentage increases to $44.93 \%$. Host plants of galls came from the following records: 209 from field sampling, 158 from herbarium specimens, and 257 from previous records in the country (Table 1). We considered our field sampling to be satisfactory since in the transects done at the end of our field study we found only four new host plant records (from 122 individuals with galls belonging to 65 species). Galls found in the field represent $17.80 \%$ of the flora in the reserve (and $23.26 \%$ of the dicots); 77 gall records from the field were also found in the herbarium and 110 were already reported in Costa Rica. It should be noted that 92 previous gall records were from La Selva Biological Station (17 km away from TBR). From the 158 gall records in the herbarium we classified 118 as almost certainly a gall and 40 as possibly galls (requiring confirmation). From the 40 doubtful records in herbarium, 21 were also found in the field or as previous records, suggesting that these doubtful records from the herbarium are probably gall hosts. It is interesting to note that the herbarium records were not homogeneous across taxa; for example, we found galls in the herbarium on all seven species of Burseraceae, but only four of the 79 species of Rubiaceae (Table 2).
All of the seven most species-rich plant families also included the greatest number of plant species with galls: Fabaceae (29), Melastomataceae (23), Rubiaceae (23), Araceae (21), Moraceae (17), Piperaceae (16), and Asteraceae (16) (Table 2). Nonetheless, most dicot families have similar proportions of gall-harboring species. The average percentage of gall-harboring species in the five most species-rich dicot families was $38.35 \%$ while for the next four most species-rich families (all with 20 or more plant species) the average was $50.98 \%$. Similarly, for the 14 dicots families with 10-19 species the percentage was 50.28 $\%$; 28 of the 43 dicot families with fewer than three species had at least one species harboring galls. Some families had a high proportion of species with galls: Bignoniaceae (10/10), Burseraceae (7/7), Chrysobalanaceae (5/5), Dilleniaceae (4/4), Nyctaginaceae (5/6), Sapindaceae (8/10), Sapotaceae (7/8) and Vochysiaceae (4/4). Similarly, most species in genera such as Mikania (Asteraceae), Inga (Fabaceae), Ficus (Moraceae), Eugenia (Myrtaceae), Neea (Nyctaginaceae), Pouteria (Sapotaceae), and Smilax (Smilacaceae) had galls, as did all species of Protium (Burseraceae), Paullinia (Sapindaceae), and Vochysia (Vochysiaceae).

With respect to the gall-inducers the vast majority of the galls were induced 
TABLE 2

Vascular plants recorded from the Tirimbina Biological Reserve (TBR), indicating (in bold) those that harbor galls

\begin{tabular}{|c|c|}
\hline Plant Family & Plant species \\
\hline ACANTHACEAE (7/19) & $\begin{array}{l}\text { Aphelandra dolichantha, } \text { A. } \text { golfodulcensis }^{\mathrm{H}}, \text { A. storkii, Bravaisia integerrima }{ }^{\mathbf{T H}}{ }^{*} \text {, } \\
\text { Herpetacanthus panamensis }{ }^{\mathbf{H}}, \text { Hygrophila costata, Justicia comata, J. pectoralis, } \\
\text { J. trichotoma, Mendoncia retusa }{ }^{\mathrm{TH}}, \text { M. tonduzii } \mathbf{H}^{*}, \text { Odontonema cuspidatum, } \\
\text { O. tubaeforme, Razisea spicata }{ }^{\mathrm{H}^{*}}, \boldsymbol{R} . \text { wilburii }^{\mathrm{T}} \text {, Ruellia bioleyii, R. blechum, } \\
\text { R. metallica, R. tubiflora. }\end{array}$ \\
\hline
\end{tabular}

ACHARIACEAE $(0 / 1)$

ACTINIDACEAE $(0 / 1)$

AMARANTHACEAE $(1 / 7)$

AMARYLLIDACEAE $(0 / 1)$

ANACARDIACEAE (1/3)

ANNONACEAE (7/15)

APOCYNACEAE (6/13)

AQUIFOLIACEAE (1/1)

ARACEAE (21/66)

ARALIACEAE (3/6)

ARECACEAE (1/27)

ARISTOLOCHIACEAE (0/3)

ASPLENIACEAE (0/8)
Carpotroche platyptera.

Saurauia yasicae.

Alternanthera costaricensis, Amaranthus dubius cf., A. spinosus, Chamissoa altissima, Cyathula achyranthoides, C. prostrata, Iresine diffusa*

Crinum erubescens.

Spondias mombin, $\mathbf{S}$. radlkoferi ${ }^{\mathrm{T}}$, Tapirira guianensis.

Anaxagorea crassipetala ${ }^{\mathrm{H}^{*}}$, Annona amazonica, A. montana, A. papilionella $^{\mathrm{H}}$, A. volubilis, Cymbopetalum torulosum, Desmopsis microcarpa $\mathbf{T H}^{\mathbf{*}}$,

Guatteria aeruginosa, G. amplifolia ${ }^{T^{*}}$, Sapranthus viridiflorus*,

Stenanona penduliflora, Unonopsis hammelii, U. pittieri ${ }^{\mathbf{H}}, X_{\text {ylopia bocatorena }}{ }^{\mathrm{T}}$, $X$. sericophylla.

Allomarkgrafia plumeriiflora, Asclepias curassavica, Aspidosperma spruceanum*, Forsteronia myriantha ${ }^{\mathrm{T}^{*}}$, Gonolobus albomarginatus, Lacmellea panamensis ${ }^{\mathrm{T}^{*}}$, Mandevilla hirsuta ${ }^{\mathrm{H}}$, Mesechites trifida, Odontadenia puncticulosa*,

Prestonia portobellensis*, Tabernaemontana donnell-smithii, T. robinsonii, Tassadia obovata.

Ilex skutchii ${ }^{\mathrm{H}}$

Anthurium acutangulum, A. bakeri", A. bradeanum, A. clavigerum, A. clidemioides, A. consobrinum ${ }^{\mathbf{T}^{*}}$, A. flexile ${ }^{\mathbf{T}}$, A. formosum, A. friedrichsthalii, A. interruptum,

A. lancifolium, A. obtusum*, A. ochranthum ${ }^{\mathrm{T}}$, A. pentaphyllum $^{\mathrm{T}}{ }^{*}$, A. prolatum,

A. ramonense, $A$. scandens ${ }^{*}$, A. spathiphyllum, A. subsignatum* ${ }^{*}$ A. trisectum,

A. upalaense $e^{*}$ Dieffenbachia grayumiana cf., D. nitidipetiolata, D. hammelii,

D. aff. oerstedii, Dracontium gigas, Heteropsis oblongifolia ${ }^{\mathbf{T}}$, Monstera adansonii,

M. dissecta $^{\mathrm{T}}$, M. glaucescens $^{\mathrm{T}}$, M. molinae, M. pittieri, M. tenuis ${ }^{*}$, M. tuberculata, Philodendron alliodorum, P. angustilobum, P. aromaticum, P. aurantiifolium ${ }^{\mathbf{T}^{*}}$,

P. davidsonii, P. fragrantissimum, $P$. grandipes, $P$. hederaceum, $P$. inaequilaterum,

P. jodavisianum, P. ligulatum ${ }^{\mathrm{T}}$, P. opacum, P. platypetiolatum ${ }^{\mathbf{T}^{*}}$, P. pterotum,

${\text { P. } \text { radiatum }^{\mathrm{T}^{*}} \text {, P. rhodoaxis, P. rigidifolium }}^{\mathrm{T}}$, P. tenue, P. tripartitum ${ }^{*}$, P. wendlandii,

Rhodospatha wendlandii, Spathiphyllum friedrichsthalii, S. fulvovirens, S. laeve,

S.phryniifolium, S. wendlandii, Stenospermation angustifolium ${ }^{\mathrm{T}}$, S. marantifolium,

Syngonium macrophyllum, S. rayi, S. schottianum, S. triphyllum*.

Dendropanax arboreus ${ }^{\mathbf{T}^{*}}$, Hydrocotyle leucocephala, H. torresiana, H. umbellata,

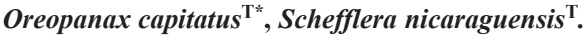

Asterogyne martiana, Astrocaryum alatum, A. confertum, Bactris caudata,

B. coloradonis, B. gracilior, B. hondurensis, B. longiseta $\mathrm{cf}$.,

Chamaedorea deckeriana, C. lucidifrons, C. pinnatifrons, C. tepejilote,

Desmoncus moorei, Euterpe precatoria, Geonoma congesta, G. cuneata $^{\mathbf{T}^{*}}$, G. deversa,

G. interrupta, G. longevaginata, Iriartea deltoidea, Pholidostachys pulchra,

Prestoea decurrens, Reinhardtia gracilis, R. simplex, Socratea exorrhiza,

Synechanthus warscewiczianus, Welfia regia.

Aristolochia constricta, A. pilosa. A. sp.

Asplenium auritum, A. cirrhatum, A. formosum, A. holophlebium, A. pteropus, A. riparium, $A$. serra, $A$. serratum. 
TABLE 2 (Continued)

\begin{tabular}{|c|c|}
\hline Plant Family & Plant species \\
\hline ASTERACEAE (16/39) & 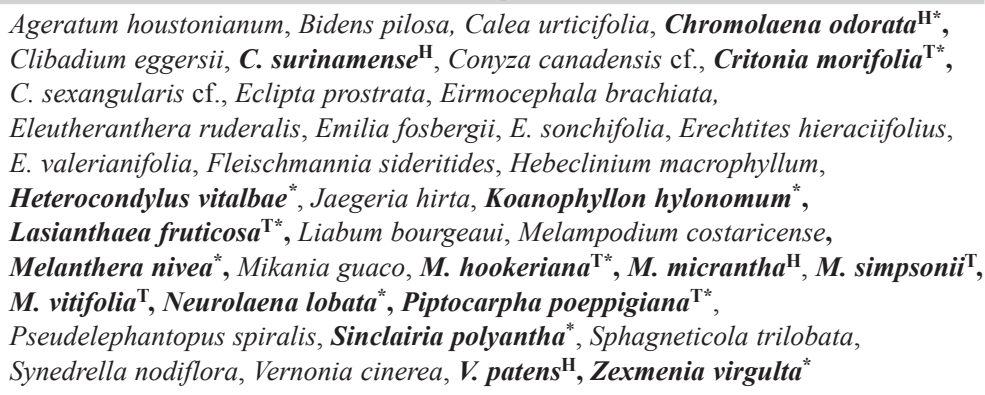 \\
\hline ATHYRIACEAE (1/6) & $\begin{array}{l}\text { Diplazium cristatum cf., D. grandifolium, D. lindbergii, D. macrophyllum }{ }^{*} \text {, } \\
\text { D. pactile cf., D. striatastrum. }\end{array}$ \\
\hline BEGONIACEAE (1/4) & Begonia glabra ${ }^{\mathbf{T}}, B$. multinervia, $B$. semiovata,$B$. sericoneura. \\
\hline BIGNONIACEAE (10/10) & 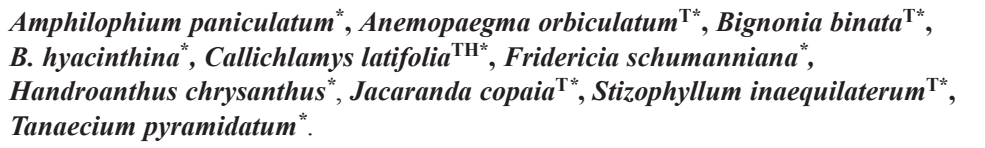 \\
\hline BLECHNACEAE (1/4) & Blechnum gracile, B. occidentale, B. polypodioides, Salpichlaena volubilis ${ }^{\mathbf{T}}$. \\
\hline BORAGINACEAE (4/9) & $\begin{array}{l}\text { Cordia alliodora }{ }^{\mathrm{TH}^{*}} \text {, C. bicolor }{ }^{*}, \text { C. cymosa, C. dwyeri, C. lucidula, C. megalantha, } \\
\text { Tournefortia angustiflora } \text { cf. }^{\mathbf{H}} \text {, Varronia dichotoma, V. spinescens }{ }^{*} .\end{array}$ \\
\hline BROMELIACEAE (1/19) & $\begin{array}{l}\text { Aechmea magdalenae, A. mariae-reginae*, A. mexicana, A. nudicaulis, A. pubescens, } \\
\text { Androlepis skinneri, Guzmania lingulata, G. monostachia, G. scherzeriana, } \\
\text { Pitcairnia arcuata, P. wendlandii, Tillandsia anceps, T. bulbosa, T. festucoides, } \\
\text { T. monadelpha, Vriesea heliconioides, Werauhia gladioliflora, W. kupperiana, } \\
\text { W. vittata. }\end{array}$ \\
\hline BURSERACEAE $(7 / 7)$ & 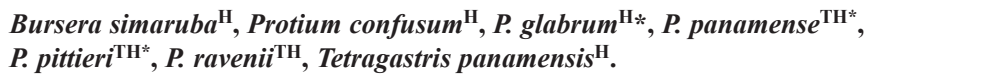 \\
\hline CACTACEAE $(1 / 6)$ & $\begin{array}{l}\text { Epiphyllum hookeri* }{ }^{*} \text { E. thomasianum, Hylocereus stenopterus, } \\
\text { Pseudorhipsalis acuminata, Rhipsalis baccifera, Weberocereus tunilla. }\end{array}$ \\
\hline CALOPHYLLACEAE $(2 / 3)$ & Calophyllum brasiliense ef. $^{\mathrm{TH}^{*}}$, Marila laxiflora, M. pluricostata $^{\mathrm{T}}$. \\
\hline CAMPANULACEAE $(0 / 1)$ & Hippobroma longiflora \\
\hline CANNABACEAE $(2 / 3)$ & Celtis iguanaea*, C. schippii, Trema micrantha* \\
\hline CAPPARACEAE $(0 / 2)$ & Capparidastrum discolor, Preslianthus pittieri. \\
\hline CARICACEAE $(0 / 2)$ & Jacaratia dolichaula, J. spinosa. \\
\hline CARYOPHYLLACEAE $(0 / 1)$ & Drymaria cordata. \\
\hline CELASTRACEAE $(0 / 2)$ & Crossopetalum parviflorum, Tontelea hondurensis. \\
\hline CHLORANTHACEAE $(0 / 1)$ & Hedyosmum scaberrimum. \\
\hline CHRYSOBALANACEAE $(5 / 5)$ & 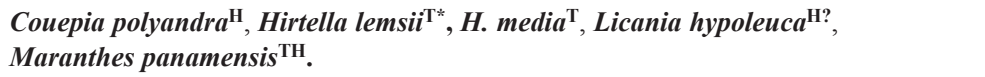 \\
\hline CLETHRACEAE $(1 / 1)$ & Clethra costaricensis ${ }^{\mathrm{TH}}$. \\
\hline CLUSIACEAE (4/8) & $\begin{array}{l}\text { Chrysochlamys nicaraguensis, C. silvicola, Clusia } \text { croatii }^{\mathrm{H} ?^{*}}, \text { C. cylindrica, } \\
\text { C. } \text { gracilis }^{\mathrm{TH}} \text {, Dystovomita paniculata } \\
\text { Symphonia globulifera* }\end{array}$ \\
\hline COMBRETACEAE $(2 / 3)$ & Combretum laxum, Terminalia amazonia ${ }^{\mathrm{T}}$, T. oblonga $^{\mathrm{TH}}$. \\
\hline COMMELINACEAE $(1 / 10)$ & $\begin{array}{l}\text { Cochliostema odoratissimum, Commelina diffusa, Dichorisandra amabilis, } \\
\text { D. hexandra, Floscopa robusta, Murdannia nudiflora, Tradescantia schippii, } \\
\text { T. zanonia*, T. zebrina, Tripogandra serrulata. }\end{array}$ \\
\hline CONNARACEAE (1/4) & Cnestidium rufescens, Connarus costaricensis, Rourea adenophora, $\boldsymbol{R}$. suerrensis ${ }^{\mathbf{T}}$. \\
\hline CONVOLVULACEAE (2/6) & $\begin{array}{l}\text { Dicranostyles ampla }{ }^{\mathbf{T}} \text {, Ipomoea alba, I. phillomega, I. tiliacea, } \\
\text { Maripa nicaraguensis }{ }^{\mathbf{T H}^{*}}, \text { Merremia tuberosa. }\end{array}$ \\
\hline
\end{tabular}


TABLE 2 (Continued)

\begin{tabular}{|c|c|}
\hline Plant Family & Plant species \\
\hline COSTACEAE $(1 / 5)$ & Costus bracteatus, C. laevis, C. lima cf $^{\mathrm{T}}$, C. malortieanus, $C$. pulverulentus. \\
\hline CUCURBITACEAE $(1 / 10)$ & $\begin{array}{l}\text { Cayaponia hammelii }{ }^{\mathbf{T}} \text {, C. prunifera, Cionosicys guabubu cf., Fevillea cordifolia, } \\
\text { Gurania coccinea, G. makoyana, Melothria dulcis, M. pendula, Psiguria triphylla, } \\
\text { Sechium pittieri. }\end{array}$ \\
\hline CYATHEACEAE $(1 / 3)$ & Alsophila cuspidata, Cyathea bicrenata, $\boldsymbol{C}$. multiflora ${ }^{\mathbf{T}}$. \\
\hline CYCLANTHACEAE $(3 / 16)$ & $\begin{array}{l}\text { Asplundia euryspatha*, A. ferruginea, A. longitepala, A. multistaminata, A. sleeperae, } \\
\text { A. uncinata, A. utilis, A. vagans, Carludovica sulcata, Chorigyne cilindrica } \mathrm{cf} ., \\
\text { Cyclanthus bipartitus, Dicranopygium umbrophilum, D. wedelii }{ }^{*}, \\
\text { Evodianthus funifer }{ }^{*}, \text { Ludovia integrifolia, Sphaeradenia acutitepala. }\end{array}$ \\
\hline CYPERACEAE (0/16) & $\begin{array}{l}\text { Cyperus esculentus cf., C. laxus, C. luzulae, C. miliifolius cf., C. odoratus, C. simplex, } \\
\text { C. thyrsiflorus, Elaeocharis elegans, E. retroflexa, Fimbristylis dichotoma, } \\
\text { Kyllinga pumila, Mapania assimilis, Rhynchospora radicans, Scleria latifolia, } \\
\text { S. melaleuca, S. secans. }\end{array}$ \\
\hline DENNSTAEDTIACEAE $(0 / 2)$ & Dennstaedtia cicutaria, Hypolepis hostilis. \\
\hline DICHAPETALACEAE (1/5) & $\begin{array}{l}\text { Dichapetalum axillare, D. nervatum }{ }^{\mathbf{T}}, \text { D. nevermannianum, D. rugosum, } \\
\text { Tapura guianensis. }\end{array}$ \\
\hline DILLENIACEAE (4/4) & $\begin{array}{l}\text { Davilla nitida }{ }^{\mathrm{T}}, \text { Doliocarpus multiflorus }^{\mathrm{T}}, \text { Pinzona } \text { coriacea }^{\mathrm{H}} \\
\text { Tetracera portobellensis }^{\mathrm{T}} \text {. }\end{array}$ \\
\hline DIOSCOREACEAE $(0 / 2)$ & Dioscorea hondurensis, D. spiculiflora. \\
\hline DRYOPTERIDACEAE $(0 / 17)$ & $\begin{array}{l}\text { Bolbitis portoricensis, Didymochlaena truncatula, Elaphoglossum correae cf., } \\
\text { E. amygdalifolium, E. herminieri, E. latifolium, E. peltatum, E. variabile cf., } \\
\text { Lastreopsis exculta, Megalastrum subincisum cf., Mickelia nicotianifolia, } \\
\text { M. oligarchica } \text { cf., Olfersia cervina, Polybotrya caudata, P. osmundacea, } \\
\text { Stigmatopteris lechleri, S. longicaudata. }\end{array}$ \\
\hline ELAEOCARPACEAE $(0 / 1)$ & Sloanea geniculata. \\
\hline ERICACEAE $(2 / 2)$ & Satyria panurensis $^{\mathrm{TH}}$, Sphyrospermum buxifolium $\mathrm{H}^{\mathrm{H}^{*}}$. \\
\hline ERYTHROXYLACEAE (1/2) & Erythroxylum fimbriatum, E. macrophyllum ${ }^{\mathbf{T H}^{*}}$ \\
\hline EUPHORBIACEAE (11/27) & 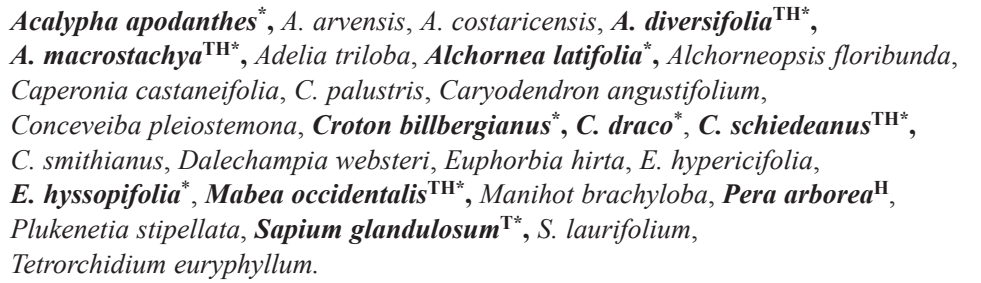 \\
\hline FABACEAE $(29 / 67)$ & 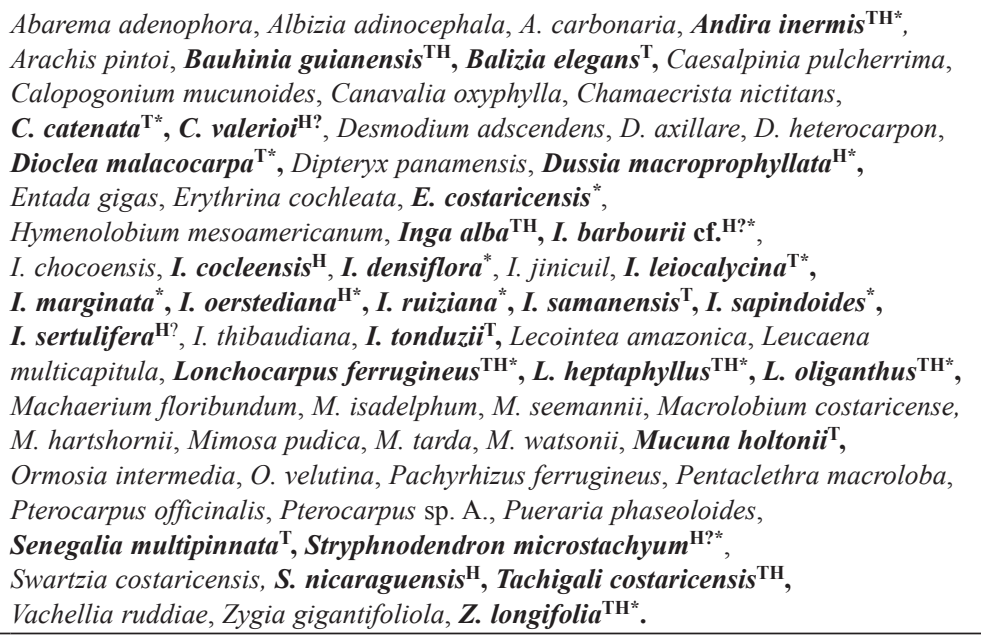 \\
\hline
\end{tabular}


TABLE 2 (Continued)

\begin{tabular}{|c|c|}
\hline Plant Family & Plant species \\
\hline GENTIANACEAE $(0 / 3)$ & Lisianthius skinneri, Potalia turbinata, Voyria tenella. \\
\hline GESNERIACEAE $(12 / 25)$ & 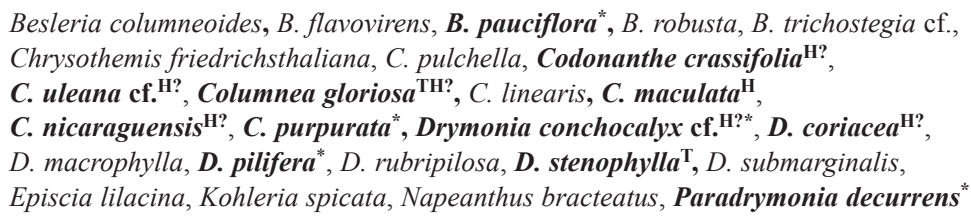 \\
\hline GLEICHENIACEAE $(0 / 1)$ & Sticherus bifidus \\
\hline GNETACEAE $(0 / 1)$ & Gnetum leyboldii \\
\hline HAEMODORACEAE $(0 / 1)$ & Xiphidium caeruleum \\
\hline HELICONIACEAE $(0 / 8)$ & $\begin{array}{l}\text { Heliconia imbricata, H. irrasa, H. latispatha, H. mariae, H. mathiasiae, } \\
\text { H. pogonantha, H. umbrophila, H. wagneriana. }\end{array}$ \\
\hline HERNANDIACEAE (2/2) & Hernandia didymantha ${ }^{\mathrm{T}^{*}}$, Sparattanthelium amazonum*". \\
\hline HUMIRIACEAE (2/2) & Humiriastrum diguense ${ }^{\mathrm{H} ?^{*}}$, Sacoglottis trichogyna ${ }^{\mathrm{T}^{*}}$. \\
\hline HYMENOPHYLLACEAE (0/13) & $\begin{array}{l}\text { Didymoglossum curtii, D. ekmanii, D. godmanii, D. membranaceum, } \\
\text { Hymenophyllum hirsutum, H. maxonii cf., Trichomanes crispum cf., T. curtii, } \\
\text { T. diaphanum, T. elegans, T. pinnatum, T. tuerckheimii, Vandenboschia collariata. }\end{array}$ \\
\hline HYPERICACEAE (0/2) & Vismia billbergiana, $V$. macrophylla \\
\hline ICACINACEAE (2/2) & Calatola costaricensis ${ }^{\mathrm{H}^{*}}$, Leretia cordata ${ }^{\mathrm{T}}$. \\
\hline LACISTEMACEAE (1/2) & Lacistema aggregatum ${ }^{\mathbf{T H}^{*}}$, Lozania pittieri. \\
\hline LAMIACEAE $(2 / 10)$ & $\begin{array}{l}\text { Aegiphila cephalophora }{ }^{*} \text {, A. elata, } \text { A. } \text { falcata }^{\mathbf{T H}^{*}} \text {, Callicarpa acuminata, } \\
\text { Hyptis capitata, H. obtusiflora, H. suaveolens, } H . \text { verticillata, H. vilis, Vitex cooperi. }\end{array}$ \\
\hline LAURACEAE (12/19) & 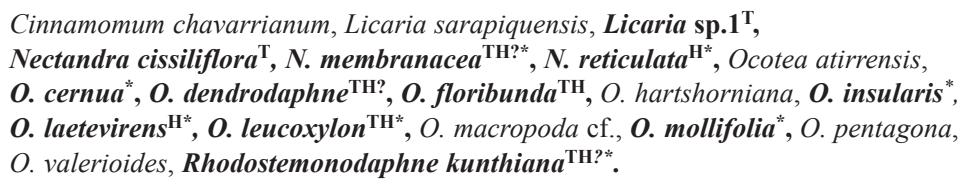 \\
\hline LECYTHIDACEAE $(0 / 1)$ & Lecythis ampla. \\
\hline LINDERNIACEAE $(0 / 3)$ & Lindernia crustacea, L. diffusa, Torenia thouarsii. \\
\hline LINDSAEACEAE $(0 / 3)$ & Lindsaea lancea, L. quadrangularis cf., Odontosoria gymnogrammoides. \\
\hline LOGANIACEAE $(0 / 2)$ & Spigelia humboldtiana, Strychnos peckii. \\
\hline LOMARIOPSIDACEAE $(0 / 2)$ & Lomariopsis japurensis, L. vestita \\
\hline LORANTHACEAE $(2 / 2)$ & Oryctanthus occidentalis ${ }^{\mathrm{H}}$, Phthirusa pyrifolia $\mathrm{cf}^{\mathrm{H}^{*}}$. \\
\hline LYCOPODIACEAE $(0 / 2)$ & Huperzia dichotoma, Lycopodiella cernua. \\
\hline LYTHRACEAE (2/4) & Cuphea carthagenensis, C. epilobiifolia ${ }^{*}$, C. hyssopifolia, C. utriculosa ${ }^{\mathbf{H}}$. \\
\hline MAGNOLIACEAE (1/1) & Talauma gloriensis ${ }^{\mathrm{H}{ }^{*}}$ \\
\hline MALPIGHIACEAE (5/12) & $\begin{array}{l}\text { Banisteriopsis elegans }{ }^{\mathbf{H} ?} \text {, Bunchosia macrophylla, B. polystachia, } \\
\text { Byrsonima arthropoda }^{\mathbf{H} ?}, \text { B. }_{\text {crassifolia }} \mathbf{H}^{*}, \text { Heteropterys macrostachya, } \\
\text { H. panamensis }{ }^{\mathrm{T}} \text {, Hiraea fagifolia }{ }^{\mathbf{T}}, \text { Jubelina wilburii, Stigmaphyllon lindenianum, } \\
\text { Tetrapterys tinifolia, T. schiedeana. }\end{array}$ \\
\hline MALVACEAE (12/23) & 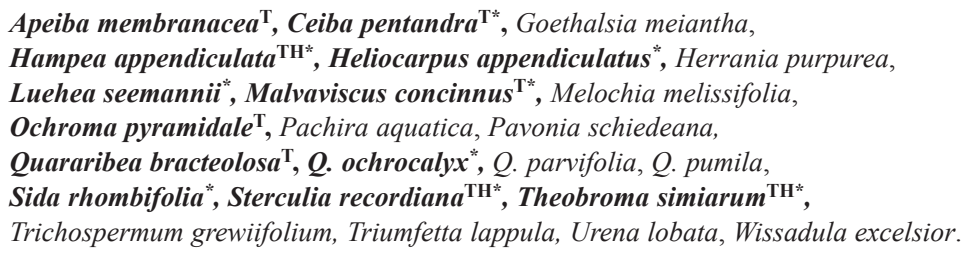 \\
\hline MARANTACEAE $(0 / 13)$ & $\begin{array}{l}\text { Calathea donnell-smithii, C. gymnocarpa, C. lasiostachya, C. leucostachys, C. lutea, } \\
\text { C. marantifolia, C. micans, C. warscewiczii, Hylaeanthe hoffmannii, } \\
\text { Ischnosiphon elegans, I. inflatus, Pleiostachya leiostachya, P. pruinosa. }\end{array}$ \\
\hline MARATTIACEAE $(0 / 3)$ & Danaea media, D. moritziana, D. wendlandii. \\
\hline
\end{tabular}


TABLE 2 (Continued)

\begin{tabular}{|c|c|}
\hline Plant Family & Plant species \\
\hline MARCGRAVIACEAE (0/5) & Marcgravia nepenthoides, M. nervosa, M. serrae, Ruyschia enervia, Souroubea gilgii. \\
\hline MELASTOMATACEAE $(23 / 57)$ & 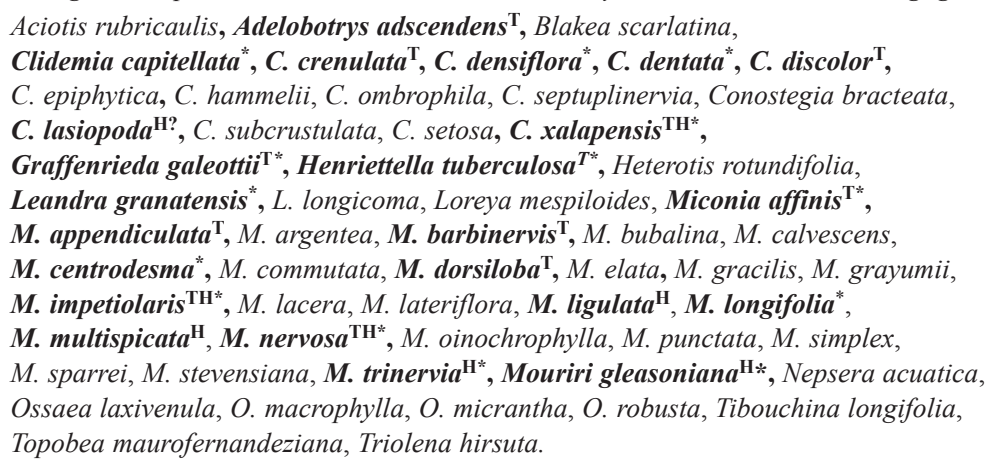 \\
\hline MELIACEAE $(6 / 11)$ & 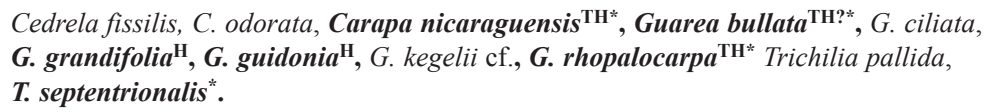 \\
\hline MENISPERMACEAE (3/8) & $\begin{array}{l}\text { Abuta panamensis }{ }^{\mathbf{T}^{*}}, \text { Anomospermum reticulatum, Cissampelos andromorpha } \\
\text { C. grandifolia, } \boldsymbol{C} \text {. pareira }{ }^{\mathbf{H}^{*}}, \text { C. tropaeolifolia, Disciphania calocarpa, } \\
\text { Odontocarya truncata. }\end{array}$ \\
\hline METAXYACEAE $(0 / 1)$ & Metaxya rostrata \\
\hline MONIMIACEAE $(0 / 1)$ & Mollinedia costaricensis. \\
\hline MORACEAE (17/27) & 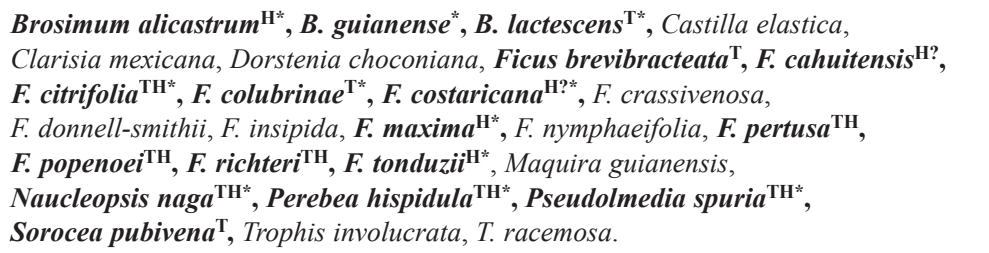 \\
\hline MYRISTICACEAE (3/4) & Compsoneura mexicana, Otoba novogranatensis ${ }^{*}$, Virola koschnyi ${ }^{\mathrm{TH}}$, V sebifera $^{\mathrm{TH}^{*}}$. \\
\hline MYRTACEAE $(4 / 7)$ & $\begin{array}{l}\text { Eugenia earthiana, } \text { E. } \text { basilaris }^{\mathbf{T}}, \text { E. hammelii, E. hartshornii }{ }^{\mathbf{T}}, \text { E. } \text { sarapiquensis }^{*} \text {, } \\
\text { Myrcia aliena, M. } \text { splendens }^{\mathbf{T}^{*}} .\end{array}$ \\
\hline NEPHROLEPIDACEAE (0/1) & Nephrolepis biserrata cf. \\
\hline NYCTAGINACEAE $(5 / 6)$ & $\begin{array}{l}\text { Neea amplifolia }{ }^{\mathrm{TH}}, N . \text { elegans }^{*}, N . \text { laetevirens }^{\mathrm{TH}^{*}}, N . \text { popenoei, }^{*} \text {. } \text { urophylla }^{\mathrm{T}} \text {, } \\
\text { Pisonia aculeata }^{\mathrm{TH} ?^{*}} \text {. }\end{array}$ \\
\hline OCHNACEAE $(2 / 5)$ & $\begin{array}{l}\text { Cespedesia spathulata, Lacunaria panamensis }{ }^{\mathrm{TH}^{*}}, \text { Ouratea crassinervia } \\
\text { Quiina macrophylla, Sauvagesia erecta. }\end{array}$ \\
\hline OLACACEAE $(1 / 2)$ & Heisteria scandens, Minquartia guianensis ${ }^{T^{*}}$ \\
\hline OLEACEAE $(0 / 1)$ & Chionanthus panamensis. \\
\hline OLEANDRACEAE $(0 / 1)$ & Oleandra articulata. \\
\hline ONAGRACEAE $(0 / 6)$ & $\begin{array}{l}\text { Ludwigia decurrens, L. erecta } \text { cf., L. hyssopifolia, L. latifolia, L. octovalvis, } \\
\text { L. peruviana. }\end{array}$ \\
\hline ORCHIDACEAE $(1 / 27)$ & $\begin{array}{l}\text { Dichaea panamensis, D. sarapiquinsis, Elleanthus cynarocephalus, } \\
\text { Epidendrum exiguum, E. hunterianum, E. isomerum, Gongora leucochila, G. unicolor, } \\
\text { Maxillaria brachybulbon, M. confusa, M. uncata, Microchilus tridax, } \\
\text { Myoxanthus colothrix, Oncidium stenotis, Palmorchis powellii, Pleurothallis pantasmi, } \\
\text { P. phyllocardioides, Prosthechea fragrans, Psygmorchis pusilla, Scaphyglottis behrii, } \\
\text { S. imbricata, Sobralia chrysostoma }, \text { S. fragans, Specklinia guanacastensis cf., } \\
\text { S. simmleriana, Trichosalpinx blaisdellii, Wullschlaegelia calcarata. }\end{array}$ \\
\hline OXALIDACEAE $(0 / 1)$ & Oxalis barrelieri. \\
\hline PAPAVERACEAE $(0 / 1)$ & Bocconia frutescens. \\
\hline
\end{tabular}


TABLE 2 (Continued)

\begin{tabular}{|c|c|}
\hline Plant Family & Plant species \\
\hline PASSIFLORACEAE (1/8) & $\begin{array}{l}\text { Passiflora ambigua, } P \text {. arbelaezii, P. biflora, P. megacoriacea, } P . \text { menispermifolia, } \\
\text { P. nitida, P. oerstedii, } P \text {. vitifolia }{ }^{\mathbf{T}^{*}} \text {. }\end{array}$ \\
\hline PENTAPHYLACACEAE $(0 / 1)$ & Freziera grisebachii. \\
\hline PHYLLANTHACEAE (3/8) & $\begin{array}{l}\text { Hieronyma alchorneoides }{ }^{\mathbf{T}^{*}}, \text { H. oblonga }{ }^{\mathbf{H}}, \text { Phyllanthus amarus, } P . \text { caroliniensis, } \\
\text { P. skutchii, P. stipulatus, } \text { P. urinaria, Richeria obovata }{ }^{\mathbf{H}} \text {. }\end{array}$ \\
\hline PHYTOLACCACEAE $(0 / 1)$ & Phytolacca rivinoides. \\
\hline PICRAMNIACEAE (0/1) & Picramnia latifolia. \\
\hline PIPERACEAE (16/37) & 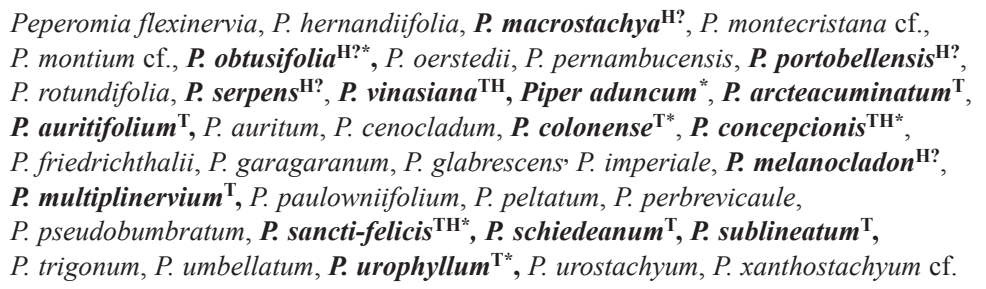 \\
\hline PLANTAGINACEAE $(0 / 2)$ & Mecardonia procumbens, Scoparia dulcis. \\
\hline POACEAE $(0 / 23)$ & $\begin{array}{l}\text { Arundinella berteroniana, Axonopus compressus, Chloris radiata, } \\
\text { Coix lacryma-jobi, Cryptochloa concinna, Echinochloa colona, } \\
\text { Elytrostachys clavigera, Gynerium sagittatum, Homolepis aturense, } \\
\text { Ichnanthus nemorosus, I. pallens, Olyra latifolia, Oplismenus burmannii, } \\
\text { Orthoclada laxa, Oryza latifolia, Panicum grande, P. polygonatum, P. trichanthum cf., } \\
\text { Paspalum conjugatum, P. fasciculatum cf., Pennisetum purpureum, } \\
\text { Rhipidocladum pacuarense cf, Streptochaeta sodiroana. }\end{array}$ \\
\hline PODOSTEMACEAE $(0 / 1)$ & Tristicha trifaria. \\
\hline POLYGALACEAE (1/3) & Moutabea gentryi ${ }^{\mathbf{T H}^{*}}$, Polygala paniculata, Securidaca diversifolia. \\
\hline POLYGONACEAE $(2 / 2)$ & Coccoloba tuerckheimii ${ }^{\mathrm{H}^{*}}$, Polygonum punctatum**. \\
\hline POLYPODIACEAE (4/16) & $\begin{array}{l}\text { Campyloneurum brevifolium }{ }^{\mathrm{T} *}, \text { C. sphenodes, Dicranoglossum panamense, } \\
\text { Microgramma lycopodioides, M. percussa, M. reptans, Niphidium oblanceolatum*, } \\
\text { Pecluma dulcis, P. hygrometrica, P. pectinata, Phlebodium pseudoaureum }{ }^{*}, \\
\text { Pleopeltis furfuracea, Serpocaulon dissimile, S. loriciforme* }{ }^{*}, \text { S. maritimum, } \\
\text { S. triseriale. }\end{array}$ \\
\hline PONTEDERIACEAE $(0 / 1)$ & Heteranthera reniformis. \\
\hline PRIMULACEAE (4/10) & $\begin{array}{l}\text { Ardisia auriculata, A. brenesii, A. fimbrillifera }{ }^{\mathbf{T}}, \text { A. opegrapha, A. pellucida, } \\
\text { A. standleyana } \mathbf{T H}^{\mathbf{T}}, \text { A. wedelii, Hymenandra pittieri } \mathbf{H}^{*}, \text { H. stenophylla, } \\
\text { Parathesis trichogyn }^{*} \text {. }\end{array}$ \\
\hline PTERIDACEAE (0/12) & $\begin{array}{l}\text { Adiantum latifolium, A.obliquum cf., A. petiolatum, Ananthacorus angustifolius, } \\
\text { Anetium citrifolium, Pityrogramma calomelanos, } P \text {. calomelanos var.austroamericana, } \\
\text { Polytaenium cajenense, P. feei, Pteris propinqua, P. pungens, Vittaria lineata. }\end{array}$ \\
\hline PUTRANJIVACEAE (1/1) & Drypetes standleyi ${ }^{\mathrm{H}^{*}}$ \\
\hline RANUNCULACEAE $(0 / 1)$ & Clematis polygama. \\
\hline RHAMNACEAE (3/4) & Colubrina spinosa $a^{\mathrm{TH}^{*}}$, Gouania hypoglauca ${ }^{\mathrm{T}}$, G. lupuloides ${ }^{\mathrm{T}}$, G. polygama. \\
\hline RHIZOPHORACEAE $(1 / 1)$ & Cassipourea elliptica $\mathrm{H}^{\mathrm{H}^{*}}$ \\
\hline
\end{tabular}


TABLE 2 (Continued)

\begin{tabular}{|c|c|}
\hline Plant Family & Plant species \\
\hline RUBIACEAE (23/79) & 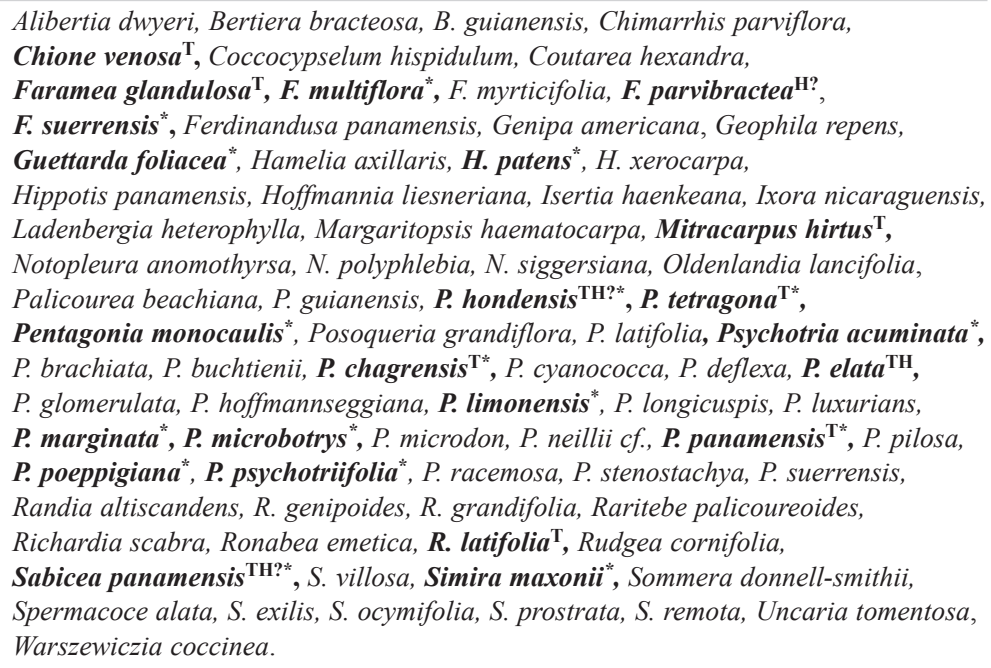 \\
\hline RUTACEAE (1/2) & Amyris brenesii, Zanthoxylum riedelianum ${ }^{\mathrm{T}}$. \\
\hline SABIACEAE $(2 / 2)$ & Meliosma donnellsmithii ${ }^{\mathrm{TH}}$, M. ${\text { glabrata } \text { cf. }^{\mathrm{T}}}^{\mathrm{T}}$ \\
\hline SACCOLOMATACEAE $(0 / 1)$ & Saccoloma inaequale. \\
\hline SALICACEAE $(5 / 10)$ & 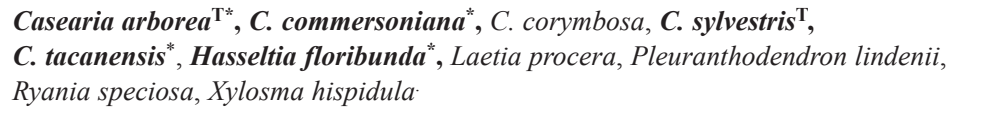 \\
\hline SAPINDACEAE (8/10) & 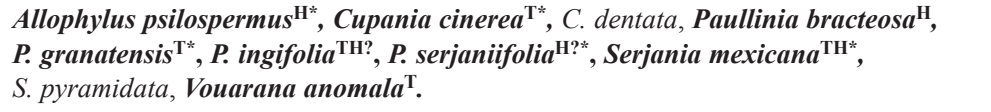 \\
\hline SAPOTACEAE (7/8) & 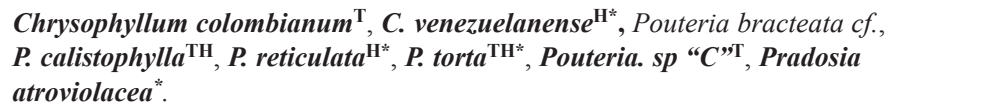 \\
\hline SCHLEGELIACEAE (1/3) & Schlegelia fastigiata, S. nicaraguensis ${ }^{\mathbf{T}}$, S. parviflora $\mathrm{cf}$. \\
\hline SELAGINELLACEAE (0/7) & $\begin{array}{l}\text { Selaginella anceps, S. arthritica, S. atirrensis, S. bombycina, S. eurynota, S. flagellata, } \\
\text { S. umbrosa. }\end{array}$ \\
\hline SIMAROUBACEAE (1/1) & Simarouba amara ${ }^{\mathrm{TH}}$ \\
\hline SIPARUNACEAE (3/4) & Siparuna cuspidata, S. grandiflora ${ }^{*}$ S. pauciflora ${ }^{\mathrm{TH}^{*}}$, S. thecaphora* \\
\hline SMILACACEAE (2/3) & Smilax domingensis ${ }^{\mathrm{T}^{*}}$, S. mollis ${ }^{\mathrm{T}^{*}}$, S. officinalis. \\
\hline SOLANACEAE (11/17) & 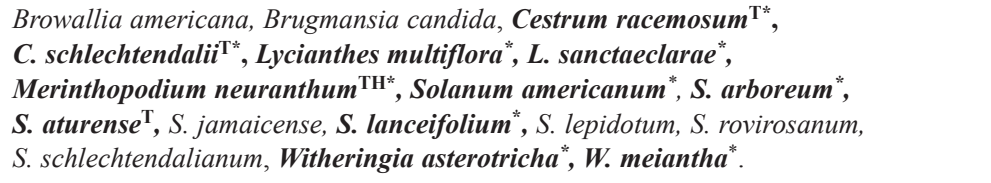 \\
\hline STAPHYLEACEAE (1/1) & Turpinia occidentalis*. \\
\hline SYMPLOCACEAE (0/1) & Symplocos striata. \\
\hline TECTARIACEAE $(0 / 6)$ & $\begin{array}{l}\text { Tectaria athyrioides, T. brauniana, T. draconoptera, T. incisa, T. nicotianifolia, } \\
\text { T. rivalis. }\end{array}$ \\
\hline THELYPTERIDACEAE $(0 / 13)$ & $\begin{array}{l}\text { Macrothelypteris torresiana, Thelypteris angustifolia, T. balbisii, T. decussata, } \\
\text { T. falcata } \text { cf., T. francoana, T. gigantea, T. hispidula } \mathrm{cf.,} \text { T. leprieurii, T. lingulata, } \\
\text { T. nicaraguensis, T. resinifera, T. serrata. }\end{array}$ \\
\hline ULMACEAE $(1 / 1)$ & Ampelocera macrocarpa ${ }^{\mathrm{T}}$. \\
\hline
\end{tabular}

RUTACEAE $(1 / 2)$
SABIACEAE $(2 / 2)$

SACCOLOMATACEAE $(0 / 1)$

SALICACEAE (5/10)

SAPINDACEAE (8/10)

SAPOTACEAE (7/8)

SCHLEGELIACEAE $(1 / 3)$

SIMAROUBACEAE (1/1)

SIPARUNACEAE (3/4)

SMILACACEAE $(2 / 3)$

SOLANACEAE (11/17)
Alibertia dwyeri, Bertiera bracteosa, B. guianensis, Chimarhis parviflora. Guettarda foliacea ${ }^{*}$, Hamelia axillaris, $\boldsymbol{H}$. patens ${ }^{*}$, H. xerocarpa,

Hippotis panamensis, Hoffmannia liesneriana, Isertia haenkeana, Ixora nicaraguensis, Ladenbergia heterophylla, Margaritopsis haematocarpa, Mitracarpus hirtus ${ }^{\mathbf{T}}$, Notopleura anomothyrsa, N. polyphlebia, N. siggersiana, Oldenlandia lancifolia, Palicourea beachiana, P. guianensis, P. hondensis ${ }^{\mathrm{TH}}$ ?* $^{\text {P. }}$ tetragona ${ }^{\mathrm{T}}$,

P. brachiata, P. buchtienii, P. chagrensis $^{\mathbf{T}}$, P. cyanococca, P. deflexa, P. elata $^{\mathbf{T H}}$, P. glomerulata, P. hoffmannseggiana, P. limonensis* ${ }^{*}$ P. longicuspis, P. luxurians, P. marginata*, P. microbotrys ${ }^{*}$, P. microdon, $P$. neillii cf., $\boldsymbol{P}$. panamensis ${ }^{\mathbf{T}}$, P. pilosa, Randia altiscandens, $R$. genipoides, $R$. grandifolia, Raritebe palicoureoides, Richardia scabra, Ronabea emetica, $R$. latifolia ${ }^{\mathbf{T}}$, Rudgea cornifolia,

Sabicea panamensis ${ }^{\text {TH?*}}$, S. villosa, Simira maxonii*, Sommera donnell-smithii, Spermacoce alata, S. exilis, S. ocymifolia, S. prostrata, S. remota, Uncaria tomentosa, Warszewiczia coccinea.

Amyris brenesii, Zanthoxylum riedelianum ${ }^{\mathrm{T}}$.

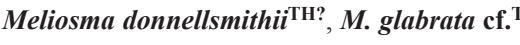

Saccoloma inaequale.

Casearia arborea ${ }^{\mathbf{T}^{*}}$, C. commersoniana*, C. corymbosa, C. sylvestris ${ }^{\mathrm{T}}$,

C. tacanensis ${ }^{*}$, Hasseltia floribunda ${ }^{*}$, Laetia procera, Pleuranthodendron lindenii, Ryania speciosa, Xylosma hispidula

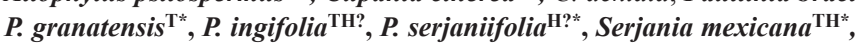
S. pyramidata, Vouarana anomala ${ }^{\mathrm{T}}$.

P. calistophylla ${ }^{\mathrm{TH}}$, P reticulata ${ }^{\mathrm{H}^{*}}$, P. torta ${ }^{\mathrm{TH}}$, Pouteria sp “ $C$ " $\mathrm{T}$, Pradosia atroviolacea*

Schlegelia fastigiata, S. nicaraguensis ${ }^{\mathbf{T}}$, S. parviflora $\mathrm{cf}$.

Selaginella anceps, S. arthritica, S. atirrensis, S. bombycina, S. eurynota, S. flagellata, S. umbrosa.

Simarouba amara ${ }^{\mathrm{TH}}$

Siparuna cuspidata, S. grandiflora ${ }^{*}$, S. pauciflora $^{\mathrm{TH}^{*}}$, S. thecaphora*

Smilax domingensis ${ }^{\mathbf{T}^{*}}, S$. mollis $^{\mathrm{T}^{*}}$, S. officinalis.

Browallia americana, Brugmansia candida, Cestrum racemosum ${ }^{\mathbf{T}^{*}}$,

C. schlechtendalii ${ }^{\mathrm{T}}$, Lycianthes multiflora ${ }^{*}$, L. sanctaeclarae ${ }^{*}$,

Merinthopodium neuranthum ${ }^{\mathrm{TH}}$, Solanum americanum ${ }^{*}$, S. arboreum ${ }^{*}$,

S. aturense ${ }^{\text {T, }}$ S. jamaicense, S. lanceifolium ${ }^{*}$, S. lepidotum, S. rovirosanum,

S. schlechtendalianum, Witheringia asterotricha*, W. meiantha*

Turpinia occidentalis*

Symplocos striata.

Tectaria athyrioides, T. brauniana, T. draconoptera, T. incisa, T. nicotianifolia, T. rivalis.

1. nicaraguensis, T. resinifera, T. serrata.

Ampelocera macrocarpa . 
TABLE 2 (Continued)

\begin{tabular}{|c|c|}
\hline Plant Family & Plant species \\
\hline URTICACEAE (7/15) & $\begin{array}{l}\text { Boehmeria aspera, Cecropia insignis }{ }^{*} \text {, C. obtusifolia }{ }^{\mathrm{T}^{*}}, \text { Coussapoa nymphaeifolia, } \\
\text { C. villosa }{ }^{*}, \text { Myriocarpa longipes, Phenax sonneratii, Pilea ecboliophylla } \\
\text { P. microphylla, P. pittieri }{ }^{*}, \text { Pourouma bicolor }{ }^{\mathrm{T}^{*}}, \text { P. minor, Urera baccifera, } \\
\text { U. lianoides }{ }^{*}, \text { U. simplex. }\end{array}$ \\
\hline VERBENACEAE (1/3) & Lantana camara $\mathbf{H}^{*}$, Stachytarpheta cayennensis, S. frantzii. \\
\hline VIOLACEAE $(1 / 2)$ & Gloeospermum boreale ${ }^{\mathbf{H}}$, Rinorea deflexiflora. \\
\hline VITACEAE $(2 / 3)$ & Cissus biformifolia, C. microcarpa ${ }^{\mathrm{T}^{*}}$, Vitis tiliifolia ${ }^{\mathrm{T}^{*}}$. \\
\hline VOCHYSIACEAE (4/4) & 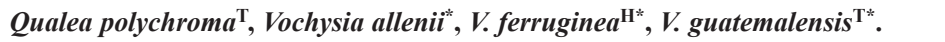 \\
\hline ZAMIACEAE $(0 / 1)$ & Zamia neurophyllidia. \\
\hline ZINGIBERACEAE (0/4) & Renealmia alpinia, $R$. cernua, $R$. concinna, $R$. pluriplicata. \\
\hline
\end{tabular}

Numbers after the plant family $=\#$ species harboring galls/total $\#$ species in TBR, $\mathrm{T}=$ galls found at TBR, asterisk $(*)=$ gall records from elsewhere in Costa Rica, $\mathrm{H}=$ gall records from the herbarium, $\mathrm{H}$ ? = gall records from the herbarium that are questionable.

by Cecidomyiidae (Diptera), while the second most common category was "gall-inducer uncertain". The latter was generally the result of galls being too young or too old, and obviously nothing can be concluded from herbarium records (but see, Veenstra, 2012). Several plant species had more than one species of gall-inducer (data to be presented elsewhere). Following are those that had only one, non-ceciodmyiid, gall inducer. Mites (Eriophyidae) were the only gall-inducers present on Acalypha macrostachya, Croton draco (Euhorbiaceae), Calatola costaricensis (Icacinaceae), Ochroma pyramidale, Quararibea bracteolosa (Malvaceae), Miconia barbinervis (Melastomataceae), and Lantana camara (Verbenaceae). Psylloidea (Hemiptera) were the only gall-inducers on Lonchocarpus ferrugineus, $L$. heptaphyllus (Fabaceae), Ocotea laetevirens, $O$. mollifolia (Lauraceae), Brosimum alicastrum, Naucleopsis naga, Pseudolmedia spuria, and Sorocea pubivena (Moraceae); Eriococcidae (Hemiptera) on Ceiba pentandra (Malvaceae); Buprestidae (Coleoptera) on Amphilophium paniculatum (Bignoniaceae); Curculionidae (Coleoptera) on Psychotria marginata (Rubiaceae); and Lepidoptera on Cuphea epilobiifolia (Lythraceae), Graffenrieda galeottii, Leandra granatensis (Melastomataceae), and Zanthoxylum riedelianum (Rutaceae). The vast majority of galls were from leaves or stems; some notable exceptions include root galls on many Araceae, and fruit galls on Geonoma cuneata (Arecaceae) and Miconia longifolia (the latter induced by a braconid wasp).

\section{DISCUSSION}

In this study we report 401 host plants of galls which represents $34.16 \%$ of the total plant species recorded in the reserve. Even when considering only plants with galls actually found in the reserve (209; i.e., excluding species for which galls were recorded only in herbarium specimens not from TBR or by previous records from elsewhere in the country), this number is higher than that reported in many previous gall inventories in the Neotropics (Cuevas-Reyes, Siebe, MartínezRamos, \& Oyama, 2003; Araújo, Silva, Santos, \& Gomes-Klein, 2013). Maia \& Mascarenhas (2017) found 432 gall morphotypes on 211 plant species in Parque Nacional do Itatiaia, Brazil, and considered this area as having the highest gall richness studied thus far in Brazil. However, it should be emphasized that gall inventories vary with respect to methodology, size of the study area, local plant diversity, and sampling effort (Dalbem \& Mendonça, 2006). Compared with most previous inventories our sampling effort was greater and was carried out for a longer period of time (three years of weekly observations), which probably explains 
the high number of galls. Several galls were found just once during the entire study period (e.g., on Philodendron ligulatum, Araceae), or only during a certain time of year (e.g., galls in infrutescences of Anthurium ochranthum, Araceae), or only in certain individuals of a plant species.

Although we found galls in the field on only $17.8 \%$ of the plant species in the reserve (but in almost $45 \%$ of the dicot species in the reserve), the actual percentage is undoubtedly much higher. The fact that we did not find galls on 147 plant species for which records exists elsewhere in Costa Rica (many of them from nearby La Selva or Braulio Carrillo National Park), strongly suggests that many of these gall-inducing arthropods are in fact present in TBR (or that some gall-inducers have a patchy distribution). Moreover, we did not sample from the canopy, which has undoubtedly resulted in our underestimating the species richness of gall-inducers in the reserve. It has been shown that the species richness of gall-inducers is higher in the canopy than in the understory (Medianero, Valderrama, \& Barrios, 2003; Ribeiro, Basset, \& Kitching, 2014). Julião et al. (2014) sampled the upper strata of 1091 trees belonging to 491 species in two reserves in Amazonia and found galls on $90 \%$ of the species.

Among dicots, large plant families generally have a greater absolute species richness of gall-inducers (Fernandes, 1992; Mendonça, 2007; Araújo, 2011; Araújo et al., 2012), a trend that is also noticeable in the results of the present study. In addition to having more species available as potential hosts of galls, large families are more likely to include abundant species and superhosts. However, by taking into account the complete plant list for the reserve, our results suggest that the proportion of plant species with galls is similar among most dicot families. The situation among monocots is probably quite different. For example, palms (Arecaceae) appear to have very few galls; the first known gall from a New World palm was recently found as a part of the present study (Gagné, Ley-López,
\& Hanson, 2018). On the other hand, families that consist primarily of epiphytes (Araceae, Bromeliaceae, Orchidaceae) probably have an underestimated species-richness of galls due to logistical difficulties in sampling. For this same reason our list of orchids in the TBR (Table 2) is very incomplete compared with that of other plant families.

Half of our records of galls were from herbarium specimens or from previous records elsewhere in the country. Given the difficulties in finding galls in the field (see Introduction), especially in wet tropical forests, these methods can be useful in supplementing gall inventories. These indirect methods, however, have limitations. Examining herbarium specimens for galls requires considerable previous experience with field-collected galls, but even with this experience, several uncertainties remain. For example, discolored leaf spots due to fungal pathogens can be easily mistaken for blister galls and we therefore excluded ambiguous cases. Even more difficult to distinguish in herbarium specimens, if not impossible, are holes in leaves caused by necrotic galls becoming detached (Fernandes et al., 2012). Some galls (e.g. those that are large or detachable) are probably underrepresented in herbarium samples, and galls hidden in flowers or fruits cannot be detected without destroying the specimen (which we did not do). Root galls are also virtually impossible to find in the field (except in exposed roots of epiphytes or aerial adventitious roots of other plants), and are very underrepresented in herbaria. Gall records for which there is only a questionable herbarium record (i.e., indicated by nothing more than $\mathrm{H}$ ? in Table 2) require field confirmation before being accepted. Nonetheless, the fact that $52.5 \%$ of our doubtful herbarium gall records were also recorded in our field sampling or from previous records in the country lends some credibility to the careful use of herbarium specimens.

As with herbarium records (which were not specimens from the TBR), records of galls collected from elsewhere in the country (indicated in Table 2 by an asterisk) are no 
guarantee that the gall is present in the TBR. There is extremely little information available on the geographic distribution of gall-inducers, especially in the tropics and especially for taxa such as Cecidomyiidae where the vast majority of species are undescribed. A gall inventory done about $400 \mathrm{~km}$ away from our study site, at Coiba National Park, Panama (Nieves-Aldrey, Ibañez, \& Medianero, 2008), identified 34 host plant species, of which 16 also occur at our study site. We found galls on ten of these plant species at the TBR, and another five are known to harbor galls elsewhere in Costa Rica (Hanson, unpublished data). More research is needed to determine how many of these shared host plant records represent identical species of gall-inducers. Arriola, Júnior, Mouga, Isaias, \& Costa (2016) provided evidence that Calophyllum brasiliense and seven cecidomyiid gall-inducers on this plant have overlapping geographical distributions. However, such information is lacking for the vast majority of gall-inducers, and although they are highly host specific, not all gall-inducers are restricted to just a single plant species. Several plant species with a wide geographic distribution have been recorded harboring galls in other countries, but not in Costa Rica, at least thus far. For example, at least four plant species present in the TBR are reported to harbor galls in Brazil, but thus far no records exist in Costa Rica: Mikania guaco, Paspalum conjugatum (Gagné, 1994), Inga thibaudiana and Lecointea amazonica (Julião et al., 2014).

In conclusion, despite the increasing number of gall inventories in the Neotropics, there is still much more work to be done, especially in the taxonomy of the gall-inducers. The results of the present investigation demonstrate the usefulness of simultaneously inventorying the plants and the galls, and of supplementing transects with longer-term, opportunistic collecting, as well as with records from nearby sites and herbaria. Deliberate inclusion of galls in herbarium samples, which is presumably not a general practice, should perhaps be encouraged since this could provide valuable information for gall researchers as well as for botanists (Veenstra, 2012; Rat \& Anačkov, 2012).

Ethical statement: authors declare that they all agree with this publication and made significant contributions; that there is no conflict of interest of any kind; and that we followed all pertinent ethical and legal procedures and requirements. All financial sources are fully and clearly stated in the acknowledgements section. A signed document has been filed in the journal archives.

\section{ACKNOWLEDGMENTS}

We gratefully acknowledge the field work of Cristian Miranda Alvarado who is responsible for a large proportion of the galls found in the field at the TBR, and to colleagues and students who have collected galls from elsewhere in the country. We give special thanks to the staff of the School of Biology of the University of Costa Rica, especially the staff of the herbarium. Carlos Garita Alvarado provided valuable suggestions that improved the original manuscript. Orlando Vargas helped in the identification of some plants and Klaus Mehltreter checked our list of ferns. Finally, we thank three anonymous reviewers for greatly improving the manuscript.

\section{RESUMEN}

Plantas y hospederos de agallas de la Reserva Biológica Tirimbina, Sarapiquí, Costa Rica: combinando muestras del campo con registros del herbario. Los estudios relacionados con la diversidad de insectos inductores de agallas han venido aumentando en el Neotrópico. Sin embargo, pocos inventarios se han realizado en sitios donde la flora está bien documentada y los registros previos de agallas, así como información disponible de herbario, han sido subutilizados. En este estudio brindamos un inventario de las plantas nativas en una reserva de 345 ha ubicada en las tierras bajas del Caribe costarricense y documentamos cuales de estas son hospederas de agallas. El muestreo de agallas se llevó a cabo entre noviembre del 2013 y diciembre del 2016. Así mismo, comparamos nuestra lista de plantas con los registros previos de agallas encontradas en el resto del país y examinamos en herbario todas las dicotiledóneas registradas en la reserva 
en busca de agallas. En total, encontramos 143 familias y 1174 especies de plantas de las cuales 401 especies fueron hospederas de agallas. Estas fueron encontradas de las siguientes categorías no mutuamente exclusivas: 209 mediante colectas de campo, 257 a partir de registros previos y 158 en material de herbario. De las plantas con agallas encontradas en el campo 77 corresponden a registros nuevos para el país, 77 fueron halladas también en herbario y 110 habían sido reportadas anteriormente en el país. El material de herbario y los registros previos demostraron ser buenos indicadores de las potenciales especies de plantas hospederas de agallas, sin embargo, alrededor de una cuarta parte (40/158) de los registros de agallas en herbario son ambiguos (difíciles de determinar si son agallas o no). El tamaño de la familia de las plantas está relacionado con la diversidad de especies de plantas hospederas de agallas, no obstante, la mayoría de las familias de dicotiledóneas tiene una proporción similar de especies hospederas de agallas. Concluimos que al menos un tercio de las especies de plantas nativas de la reserva son hospederas de agallas, pero este número incrementa a $44.93 \%$ cuando solo se consideran las dicotiledóneas. Nuestros resultados demuestran la importancia de proveer inventarios completos de plantas cuando se realizan inventarios de agallas, así como la utilidad de examinar material de herbario y contrastar registros previos para inferir la diversidad de agallas en un sitio.

Palabras clave: Cecidomyiidae; lista de especies; composición florística; diversidad de agallas; inventarios

\section{REFERENCES}

Araújo, W. S. (2011). Size, age and composition: characteristics of plant taxa as predictors of the diversity of gall-midges (Diptera: Cecidomyiidae). Revista de Biología Tropical, 59(4), 1599-1607.

Araújo, W. S., Santos, B. B., \& Gomes-Klein, V. L. (2012). Relationship between host plant diversity and gallinducing insect's richness in the Brazilian Cerrado. Neotropical Biology and Conservation, 7(1), 41-47.

Araújo, W. S. D., Silva, I. P. A., Santos, B. B. D., \& GomesKlein, V. L. (2013). Host plants of insect-induced galls in areas of cerrado in the state of Goiás, Brazil. Acta Botanica Brasilica, 27(3), 537-542.

Arriola, I. A. A., Junior, J. C. F. M., Mouga, D. M. D. S., Isaias, R. M. S., \& Costa, E. C. (2016). Where host plant goes, galls go too: new records of the Neotropical galling Cecidomyiidae (Diptera) associated with Calophyllum brasiliense Cambess. (Calophyllaceae). Checklist, 12(4), 1924.

Butterill, P. T. \& Novotny, V. (2015). Gall-forming insects in a lowland tropical rainforest: low species diversity in an extremely specialized guild. Ecological Entomology, 40, 409-419.
Carneiro, M. A. A., Branco, C. S. A., Braga, C. E. D., Almada, E. D., Costa, M. B. M., Maia, V. C., \& Fernandes, G. W. (2009). Are gall midge species (Diptera, Cecidomyiidae) host-plant specialists? Revista Brasileira de Entomologia, 53(3), 365-378.

Carneiro, R. G. S., Pacheco, P., \& Isaias, R. M. S. (2015). Could the extended phenotype extend to the cellular and subcellular levels in insect-induced galls? PLoS ONE, 10(6), e0129331.

Cuevas-Reyes, P., Siebe, C., Martínez-Ramos, M., \& Oyama, K. (2003). Species richness of gall-forming insects in a tropical rain forest: correlations with plant diversity and soil fertility. Biodiversity and Conservation, 12, 411-422.

Dalbem, R. V. \& Mendonça, M. D. S. (2006). Diversity of galling arthropods and host plants in a subtropical forest of Porto Alegre, Southern Brazil. Neotropical Entomology, 35(5), 616-624.

Espírito-Santo, M. M. \& Fernandes, G. W. (2007). How many species of gall-inducing insects are there on Earth and where are they? Annals of the Entomological Society of America, 100(2), 95-99.

Fernandes, G. W. (1992). Plant family size and age effects on insular gall-forming species richness. Global Ecology and Biogeography Letters, 2(3), 71-74.

Fernandes, G. W., Carneiro, M. A. A., Lara, A. C. F., Allain, L. R., Andrade, G. I., Julião, G. R., ... \& Silva, I. M. (1996). Galling insects on neotropical species of Baccharis (Asteraceae). Tropical Zoology, 9(3), 315-332.

Fernandes, G. W., Coelho, M. S., \& Santos, J. C. (2014). Neotropical insect galls: status of knowledge and perspectives. In G. W. Fernandes \& J. C. Santos (Eds.), Neotropical insect galls (pp. 1-14). Washington DC, USA: Springer.

Fernandes, G. W., Santos, J. C., \& Gomes, V. M. (2012). Misleading herbivory in a tropical tree. Arthopod-Plant Interactions, 6(4), 649-654.

Gagné, R. J. (1994). The gall midges of the Neotropical region. New York, USA: Cornell University Press.

Gagné, R. J., Ley-López, J. M., \& Hanson, P. E. (2018). First New World record of a gall midge from palms: a new species of Contarinia (Diptera: Cecidomyiidae) from Geonoma cuneata in Costa Rica. Proceedings of the Entomological Society of Washington, 120(1), 51-61.

Hammel, B.E., Grayum, M.H., Herrera, C. \& Zamora, N. (Eds.). (2003-2015). Manual de Plantas de Costa Rica (Vols. 1-3, 5-8). St. Louis: Missouri Bot. Garden/INBio/Museo Nacional de Costa Rica.

Hanson, P. E. \& Gómez-Laurito, J. (2005). Diversity of gall-inducing arthropods of Costa Rica. In A. Raman, C. W. Schaefer, \& T. M. Withers (Eds.), Biology, 
ecology, and evolution of gall-inducing arthropo$d s$ (pp. 673-692). New Hampshire, USA: Science Publishers.

Holdridge, L. R. (1978). Ecología basada en zonas de vida. San Jose, Costa Rica: IICA.

Isaias, R. M. S., Carneiro, R. G. S., Oliveira, D. C., \& Santos, J. C. (2013). Illustrated and annotated checklist of Brazilian gall morphotypes. Neotropical Entomology, 42, 230-239.

Julião, G. R., Venticinque, E. M., Fernandes, G. W., \& Price, P. W. (2014). Unexpected high diversity of galling insects in the Amazonian upper canopy: The savanna out there. PLoS ONE, 9(12), e114986.

Maia, C. M. \& Mascarenhas, B. (2017). Insect galls of the Parque Nacional do Itatiaia (Southeast Region, Brazil). Annals of the Brazilian Academy of Sciences, 89(1), 505-575.

Medianero, E., Valderrama, A., \& Barrios, H. (2003). Diversidad de insectos minadores de hojas y formadores de agallas en el dosel y sotobosque del bosque tropical. Acta Zoológica Mexicana, 89, 153-168.

Mendonça, M. S. (2007). Plant diversity and galling arthropod diversity - searching for taxonomic patterns in an animal-plant interaction in the Neotropics. Boletin de la Sociedad Argentina de Botánica, 42(3-4), 347-357.

Nieves-Aldrey, J. L., Ibañez, A., \& Medianero, E. (2008). Richness and composition of gall-inducing arthropods at Coiba National Park, Panama. Revista de Biología Tropical, 56(3), 1269-1286.

Oliveira, D. C., Isaias, R. M. S., Fernandes, G. W., Ferreira, B. B., Carneiro, R. G. S., \& Fuzaro, L. (2016). Manipulation of host plant cells and tissues by gall-inducing insects and adaptive strategies used by different feeding guilds. Journal of Insect Physiology, 84, 103-113.
Raman, A., Schaefer, C. W., \& Withers, T. M. (2005). Biology, Ecology, and Evolution of Gall-inducing Arthropods (Vol. 1 and 2). New Hampshire: Science Publishers Inc.

Rat, M. M., \& Anačkov, G. T. (2012). Herbarium BUNS. Plant gall collection. Zbornik Matice srpske za prirodne nauke, 122(122), 36-71.

Ribeiro, S. P., Basset, Y., \& Kitching, R. (2014). Density of Insect Galls in the Forest Understorey and Canopy: Neotropical, Gondwana or Global Patterns? In G. W. Fernandes \& J. C. Santos (Eds.), Neotropical insect galls (pp. 129-141). Washington DC, USA: Springer.

Santos, M. G., Hanson, P., Maia, V. C., \& Mehltreter, K. (2019). A review of galls on ferns and lycophytes. Environmental Entomology, 48(1), 53-60.

Shorthouse, J. D., Wool, D., \& Raman, A. (2005). Gallinducing insects - Nature's most sophisticated herbivores. Basic and Applied Ecology, 6, 407-411.

Stuart, J. J., Chen, M.-S., Shukle, R., \& Harris M. O. (2012). Gall midges (Hessian flies) as plant pathogens. Annual Review of Phytopathology, 50, 339-357.

The Taxonomic Name Resolution Service. (2018). iPlant Collaborative (Version 4.0). Retrieved from http:// tnrs.iplantcollaborative.org

Tropicos. (2018). Missouri Botanical Garden, St. Louis, $M O, U S A$. Retrieved from http://www.tropicos.org

USDA. (2018). The PLANTS Database. National Plant Data Team, Greensboro, NC, USA. Retrieved from http://plants.usda.gov

Veenstra, A. (2012). Herbarium collections - An invaluable resource for gall midge taxonomists. Muelleria, 30(1), 59-64. 